\title{
Evaluación de la no adherencia al tratamiento hipoglucemiante en la farmacia comunitaria
}

\author{
Andrea Leites-Docío', Patricia García-Rodríguez ${ }^{1,2}$, Marta Fernández-Cordeiro ${ }^{1,2}$, Lorena Tenorio-Salgueiro ${ }^{1,2}$, \\ José A. Fornos-Pérez ${ }^{1,2,3}$, N. Floro Andrés-Rodríguez ${ }^{2,4}$ \\ 1. Farmacéutico comunitario. Farmacia Fornos. Cangas do Morrazo (Pontevedra). 2. Grupo Berbés de Investigación y Docencia. 3. Profesor Asociado de \\ la Facultad de Farmacia. Universidad de Santiago de Compostela. 4. Farmacéutico comunitario. Farmacia Andrés CB. Vigo.
}

\section{PALABRAS CLAVE}

Farmacia comunitaria, adherencia, diabetes mellitus tipo 2, cuestionario MMAS-8, factores relacionados con la adherencia

\section{ABREVIATURAS}

AEMPS: Agencia Española de Medicamentos y Productos Sanitarios DE: desviación estándar DM2: diabetes mellitus tipo 2 EPA-OD: estudio postautorización otro diseño

FC: farmacia comunitaria

iDPP-4: inhibidores de la dipeptidyl peptidase 4

LOPD: Ley de Protección de Datos MMAS-8: Morisky Medication Adherence Scale 8-items OMS: Organización Mundial de la Salud

PRM: problema relacionado con los medicamentos

RNM: resultado negativo de la medicación

\section{KEYWORDS}

Community pharmacy, adherence, diabetes mellitus type 2, MMAS-8 scale, factors related to adherence

\section{RESUMEN}

Introducción: La adherencia al tratamiento en enfermedades crónicas como la diabetes mellitus (DM) es de vital importancia. El incumplimiento está directamente implicado en el aumento del número de medicamentos prescritos en enfermos crónicos y es una de las causas más importantes de fracaso de los tratamientos.

Objetivos: Evaluar en la farmacia comunitaria la prevalencia de no adherencia al tratamiento en DM tipo 2 mediante la realización del cuestionario MMAS-8.

Material y métodos: Estudio observacional transversal realizado en mayo y junio de 2018 Se seleccionaron pacientes mayores de 45 años, en tratamiento con hipoglucemiantes. Se midió la adherencia farmacoterapéutica mediante la administración del cuestionario (MMAS-8) según la puntuación obtenida en el test y la percepción que tienen sobre su tratamiento.

Resultados: Participaron 64 pacientes, 29 (45,3\%) mujeres y $35(54,7)$ hombres. Las mujeres tenían 65,5 años de edad media $(\mathrm{DE}=15,3)$ y los hombres 65,6 años $(\mathrm{DE}=12,3)$. La puntuación media del test de MMAS-8 fue de 6,2 $\pm 2,1$, no encontrando diferencias significativas entre sexos. Se encontró que el número de pacientes con baja adherencia fueron $21(32,8 \%)$, con media adherencia $19(29,7 \%)$ y con alta adherencia $24(37,5 \%)$. No se encontró relación entre el porcentaje de adherencia al tratamiento con el sexo, edad, nivel de estudios y condición laboral. La creencia de que el tratamiento mejora la enfermedad y que los medicamentos prescritos son los adecuados aumenta la adherencia.

Conclusiones: El cuestionario MMAS-8 se ha mostrado como una herramienta rápida y sencilla para la evaluación de la adherencia del tratamiento en la farmacia comunitaria.

\section{Evaluation of non-adherence to hipoglucemiary treatment in community pharmacy}

\section{ABSTRACT}

Background: The treatment adherence in chronic diseases such as diabetes mellitus is of vital importance. Noncompliance is directly implicated in the increase of the prescribed drugs number in chronic patients and is one of the most important causes of treatment failure.

Objectives: To evaluate in the community pharmacy the prevalence of non-adherence to treatment in type 2 diabetes mellitus by completing the MMAS-8 questionnaire.

Methods: Cross-sectional observational study conducted in May and June 2018. Patients older than 45 years were selected, in treatment with hypoglycemic agents. Pharmacotherapeutic adherence was measured by administering the questionnaire (MMAS-8) according to the score obtained in the test and the perception they have of their treatment.

Results: 64 patients participated, 29 (45.3\%) women and 35 (54.7) men. Women ware 65.5 years of age $(S D=15.3)$ and men 65.6 years $(S D=12.3)$. The mean score of the MMAS-8 test was $6.2 \pm 2.1$, not finding significant differences between sexes. It was found that the number of patients with low adherence was $21(32.8 \%)$, with medium adherence $19(29.7 \%)$ and with high adherence $24(37.5 \%)$. No relation was found between the percentage of adherence to treatment with sex, age, level of education and work condition. The belief that the treatment improves the disease and that the prescribed medications are adequate, increases adherence.

Conclusions: The MMAS-8 questionnaire has been shown as a quick and simple tool for evaluating the adherence of the treatment in the community pharmacy.

El estudio que se presenta formó parte del trabajo de Fin de Grado de Farmacia de la primera autora en la Facultad de Farmacia de la Universidad de Santiago de Compostela.

Recibido: 24-1-2019

Aceptado: 7-3-2019

Disponible online: 29-3-2019
Financiación: ninguna.

Conflicto de intereses: ninguno.

Cite este artículo como: Leites-Docio A, Garcia-Rodriguez P, Fernández-Cordeiro M, Tenorio-Salgueiro L, Fornos-Pérez $J A$, Andrés-Rodriguez NF. Evaluación de la no adherencia al tratamiento hipoglucemiante en la farmacia comunitaria. Farmacéuticos Comunitarios. 2019 Mar 29; 11(1):5-13. doi:10.5672/FC.2173-9218.(2019/Vol11).001.02

Correspondencia: N. Floro Andrés Rodriguez (farmaciaalaxe@galicia.com).

ISSN 1885-8619 @ SEFAC (Sociedad Española de Farmacia Familiar y Comunitaria). Todos los derechos reservados. 


\section{Introducción}

El incumplimiento terapéutico es una de las causas más habituales de los problemas relacionados con los medicamentos (PRM) y tiene consecuencias tanto clínicas en resultados negativos de la medicación (RNM) como económicas [1-3]. Está directamente implicado con el aumento en el número de medicamentos prescritos en enfermos crónicos y es una de las causas más importantes de fracaso de los tratamientos [2,4].

Por su frecuencia y repercusión, es mayor en los tratamientos crónicos, pero también afecta a los tratamientos agudos [5]. En España, la falta de adherencia a los tratamientos condiciona hasta un tercio de todos los ingresos hospitalarios y es la principal causa de descompensación e ingreso hospitalario en la insuficiencia cardiaca [6,7]. Respecto al coste económico, es muy elevado; en Estados Unidos se estima un gasto anual de 100 mil millones de dólares asociado a este motivo [8], correspondiente a ingresos hospitalarios, pérdida de productividad y muerte prematura. En los tratamientos crónicos, el incumplimiento varía desde el 10 al 80\% [5,9].

Existen diferentes grupos de factores que pueden derivar en un problema de adherencia como pueden ser el paciente, el fármaco, la enfermedad y la relación con el personal sanitario $[6,8,10,11]$. De todos ellos, algunos poseen especial relevancia como falta de conocimiento de la enfermedad, del régimen terapéutico y de las consecuencias del incumplimiento por parte del paciente; larga duración del tratamiento como en enfermedades crónicas, inadecuado seguimiento, enfermedad asintomática, presencia de efectos adversos, coste de la medicación y/o copago, no acudir a las citas médicas, presencia de dificultades cognitivas, etc. $[8,10]$.

Más del 90\% de los pacientes diabéticos lo son de tipo 2 (DM2). Es una de las principales causas de morbimortalidad prematura en países desarrollados por su capacidad de desencadenar complicaciones microvasculares (retinopatía, nefropatía y neuropatía) y macrovasculares (cardiopatía isquémica, accidente cerebrovascular e isquemia arterial periférica). Su prevalencia está aumentando alarmantemente y produce 4,8 millones de muertes al año debidas a complicaciones de la enfermedad [12]. El gasto sanitario mundial en diabetes se estimó en 673 millones de dólares en 2015, esperándose que en 2040 dicha cantidad alcance valores de 802 millones [13].

Las tasas de adherencia varían en función del tipo de diabetes, edad, sexo, complejidad del régimen de tratamiento, etc. [14-16]. Los copagos más altos, el mal uso de las plumas de insulina y la aparición de efectos adversos son factores que pronostican un incumplimiento mayor [16].

La adherencia en pacientes que toman antidiabéticos orales se encuentra entre 36 y 93\% en aquellos que llevan con el tratamiento de 6 meses a dos años, y cerca del 63\% en los tratados con insulina $[15,17]$. Tanto que Kirkman y Cramer afirman en sus estudios [15,17] que el cumplimiento terapéutico se hace más consistente en pacientes de edades avanzadas, hombres con estudios, nivel de ingresos elevado y que padezcan otras enfermedades crónicas. Un mayor cumplimiento se asocia con mejor control glucémico, reducción de aparición de comorbilidades $\mathrm{y}$, por consiguiente, un menor número de visitas al médico u hospitalizaciones [16].

Desde la farmacia comunitaria se llevan a cabo acciones para mejorar el cumplimiento terapéutico de pacientes en numerosas patologías crónicas como la diabetes [9] enfermedades cardiovasculares [18], osteoporosis [19], etc. Para conseguir una buena adherencia terapéutica el paciente debe salir de la farmacia sabiendo cómo utilizarlos adecuadamente. También es importante informarle sobre su enfermedad, la importancia del tratamiento, explicarle el correcto uso de las formas farmacéuticas, educar sobre el uso racional de los medicamentos y realizar un seguimiento farmacoterapéutico [6]. La farmacia comunitaria es el lugar idóneo para llevar a cabo intervenciones para mejorar el cumplimiento, ya que el farmacéutico es el profesional sanitario de mayor contacto con la población. Un paciente polimedicado acude a la farmacia cada 15 días a retirar su medicación, mientras que un paciente sin enfermedades crónicas acude a consulta cada 3,6 o 12 meses [9].

Debido a su experiencia en farmacoterapia y su accesibilidad en la comunidad, los farmacéuticos comunitarios pueden establecer relaciones sólidas con los pacientes y convertirse en una fuente confiable de información. Por lo tanto, se encuentran en una posición ideal para proporcionar educación al paciente, vigilar y promover el cumplimiento de los regímenes de autocuidado y terapéuticos. Además, debido a su conocimiento científico y tecnológico, están especialmente alertados sobre ciertos aspectos, como la aparición de reacciones e interacciones farmacológicas adversas y características específicas asociadas con el envejecimiento y las comorbilidades [13].

Para poder llevar a cabo programas de mejora de su adherencia, es importante conocer el porcentaje de incumplimiento y sus causas. En el caso de enfermos de diabetes, no existía hasta ahora un cuestionario específico para valorar el cumplimiento terapéutico y el que existe nunca ha sido utilizado en farmacia comunitaria [20]. Es por eso que nos hemos planteado evaluar su uso en farmacia comunitaria.

\section{Objetivos}

Objetivo general

Realizar un estudio piloto para comprobar la funcionalidad de un cuestionario de no adherencia terapéutica en diabetes en farmacia comunitaria evaluando la prevalencia resultante mediante entrevista clínica con el paciente.

\section{Objetivos específicos}

- Evaluar la utilización del cuestionario MMAS-8 en la farmacia comunitaria.

- Medir la adherencia en los tratamientos hipoglucemiantes.

- Identificar factores relacionados con la no adherencia.

- Conocer la percepción de los pacientes sobre su tratamiento.

\section{Material y métodos}

\section{Diseño del estudio}

Estudio observacional transversal clasificado por la AEMPS como Estudio posautorización con otros diseños diferentes al de seguimiento prospectivo (EPA-OD), realizado en una farmacia comunitaria de Cangas do Morrazo (Pontevedra) durante los meses de mayo y junio de 2018.

\section{Pacientes}

Población diana: usuarios del servicio de dispensación de medicamentos 
que solicitan la dispensación de su tratamiento hipoglucemiante y cumplían los siguientes criterios:

Criterios de inclusión: usuarios de la farmacia mayores de 45 años con más de dos meses en tratamiento antidiabético y que aceptaron participar en el estudio dando su consentimiento escrito.

Criterios de exclusión: menores de 45 años y mayores de 45 cuya situación psicopatológica o social pudiera dificultar la comprensión de los cuestionarios o que no aceptaron participar firmando el consentimiento informado.

\section{Cálculo del tamaño muestral}

Para conseguir una precisión del 2,0\% en la estimación de una proporción mediante un intervalo de confianza asintótico Normal con corrección para poblaciones finitas al 95\% bilateral sería necesario incluir 74 unidades experimentales en el estudio, asumiendo que la proporción esperada es del 50,0\% y que el tamaño total de la población de Cangas mayor de 45 años es de 11.660 habitantes y que la proporción de diabéticos sería [21] de 1.515, de los cuales los diagnosticados y con tratamiento de diabetes serían [21] 758, correspondiendo teóricamente, al ser 10 farmacias en el ayuntamiento, 76 por cada farmacia de dicha población.

\section{Variables}

\section{Variable principal}

Adherencia farmacoterapéutica (medida con la puntuación del cuestionario MMAS-8, modificación del test inicial de Morisky-Green-Levine) [20].

Este cuestionario está compuesto por 8 ítems de respuesta categórica Sí o NO para siete de ellos, puntuando NO igual a uno y Sí correspondiente a cero. En la quinta cuestión la puntuación es inversa, pues el SÍ es igual a uno y NO igual a cero. El último ítem utiliza una escala Likert de 5 puntos que puede tomar uno de los 5 valores $(1 ; 0,8 ; 0,6 ; 0,4 ; 0)$. Las puntuaciones obtenidas en el rango MMAS-8 oscilan entre 0 y 8 , con valores de $<6$ (baja adherencia), 6-8 (media adherencia) y $>8$ (alta adherencia). Se consideraron no adherentes aquellos individuos cuya puntuación fue menor a 6 y adherentes los que tuvieron una puntuación igual o superior a 6 .
Otras variables

- Edad: años, media (DE).

- Sexo: mujer/hombre.

- Estudios: sin estudios/primarios/secundarios/universitarios

- Situación laboral: pensionista/activo.

- Enfermedades: número y tipo.

- Medicamentos para la diabetes: prescritos (número y tipo).

- Otros medicamentos: número y tipo.

- Percepción sobre el tratamiento (preguntas 9 y 10 del cuestionario): positiva o negativa.

\section{Tratamiento estadístico}

Se utilizó el programa estadístico SPSS ${ }^{\circledR} 22.0$ para Windows ${ }^{\circledR}$. Los datos cualitativos se expresan como porcentajes y los cuantitativos como media \pm desviación estándar. Se calculó la media, desviación estándar e IC95\% de la puntuación total del cuestionario MMAS-8 para el conjunto de la muestra, para mujeres y hombres, variables cuantitativas. Se calcularon las frecuencias relativas para cada una de las demás variables categóricas del cuestionario, para el conjunto de la muestra, para hombres y para mujeres, y edad.

Se utilizaron los test de chi-cuadrado o de Fisher para el análisis de variables cualitativas, $t$ de Student para variables cuantitativas con distribución normal y Mann-Whitney para variables cuantitativas con distribución no normal. La significación estadística se fijó en $\mathrm{p}<0,05$.

\section{Consideraciones éticas}

El estudio se desarrolló de acuerdo con las normas de Buena Práctica Clínica de la Conferencia Internacional sobre Armonización (ICH E6) para un estudio de estas características. El protocolo de investigación fue aprobado por el Comité Ético de Investigación Clínica de Galicia, obteniendo su aprobación.

Confidencialidad de la información: los farmacéuticos cumplieron con las medidas de seguridad de nivel alto; es decir, cumplieron con lo establecido por la Ley de Protección de Datos para ficheros de alto nivel de seguridad (LOPD).

Los datos recogidos fueron sometidos a un proceso de codificación y disociación, de tal forma que éstos nunca pudiesen ser datos identificativos o identificables de los pacientes.

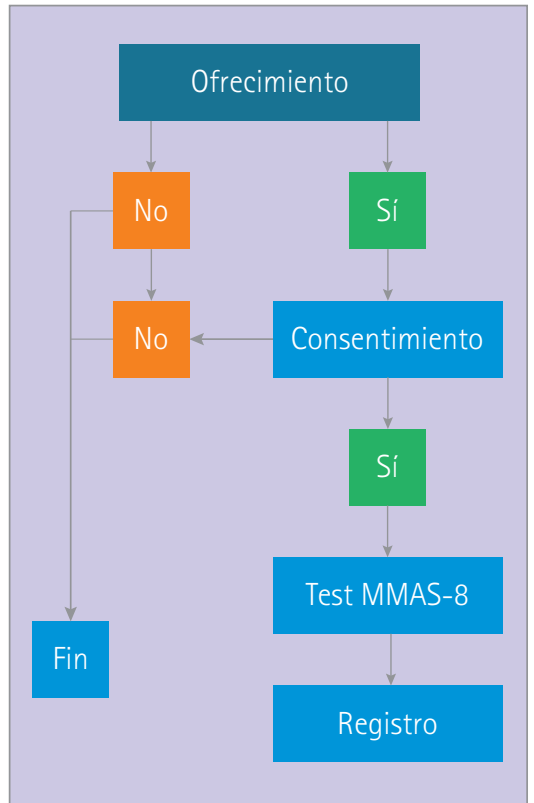

Figura 1 Esquema del estudio

Consentimiento informado: previamente a la participación en el estudio, el investigador informó adecuadamente a los pacientes, de forma verbal y escrita, del propósito y características del estudio. Se les indicó que su participación es libre y voluntaria, asegurándoles la absoluta confidencialidad de los datos y recabando su consentimiento escrito.

\section{Procedimiento (figura 1)}

Formación de los farmacéuticos: en el estudio participaron los tres farmacéuticos que ejercen en la FC donde se desarrolló el estudio. Se realizaron sesiones informativas sobre cómo realizar las entrevistas con los pacientes, la administración del cuestionario y el registro de las variables del estudio.

Ofrecimiento y selección de usuarios: con el fin de aleatorizar la incorporación al estudio se propuso la participación, durante los meses de mayo y junio de 2018, al primer paciente que acudía a la farmacia por la mañana y por la tarde y cumplía los criterios de inclusión.

Realización del test de MoriskyGreen-Levine modificado (MMAS-8): a los pacientes que aceptaron participar en el estudio y firmaron el consentimiento informado se les administró el cuestionario de adherencia a la medicación MMAS-8 y también las preguntas sobre la percepción sobre su tratamiento. Se registraron con el resto de datos incluidos en la hoja de registro (figura 2). 


\section{grupo \\ berbés}

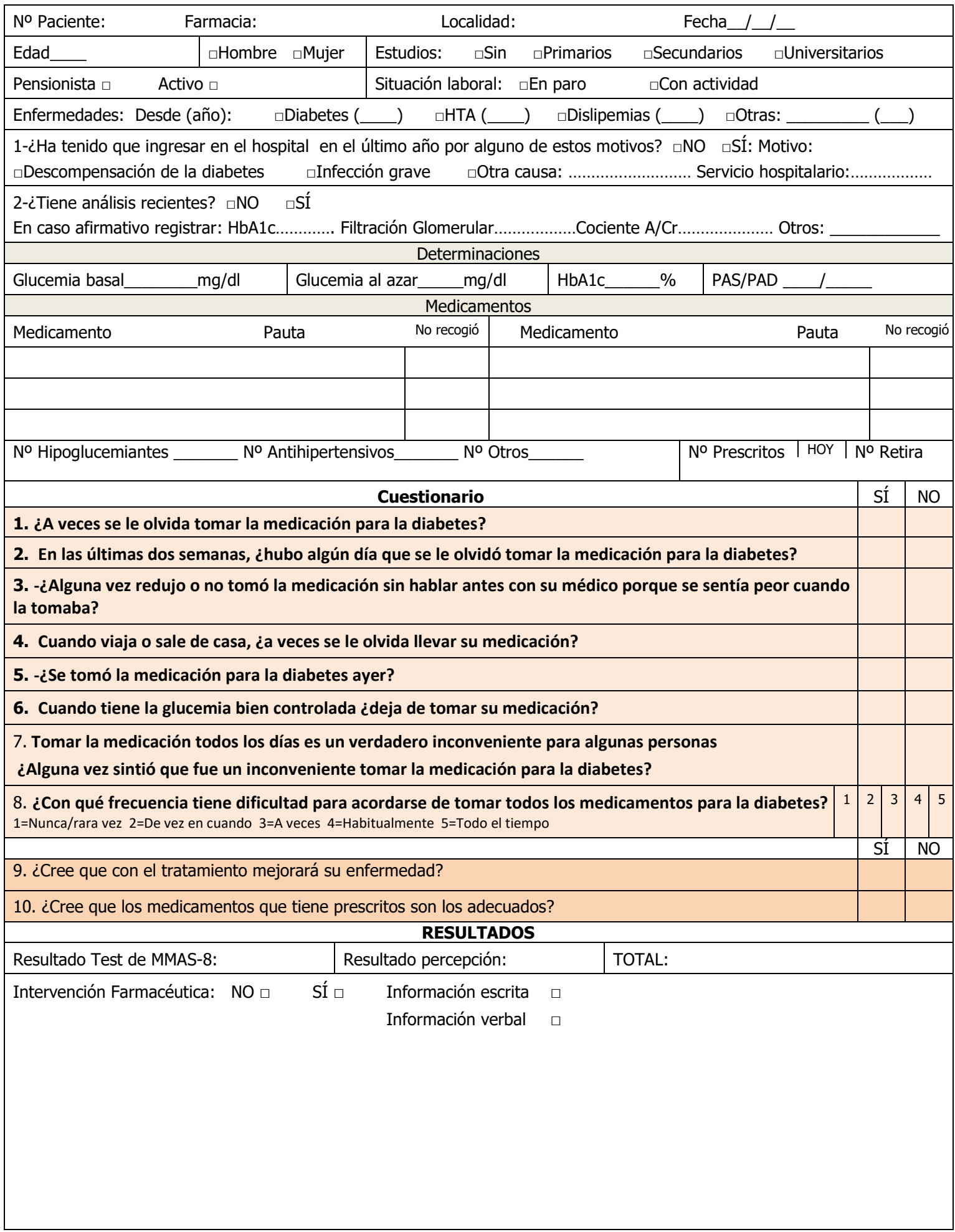

Figura 2 Hoja de registro con el cuestionario de adherencia a la medicación MMAS-8 
A todos los pacientes, tras analizar la medicación utilizada y el nivel de adherencia resultante del cuestionario, se les proporcionó de forma verbal y escrita información sobre la importancia del correcto cumplimiento de las pautas prescritas por el médico y de los servicios que el farmacéutico comunitario puede ofrecerle para conseguirlo, aunque en este pilotaje no se evaluaron los resultados de la intervención.

\section{Resultados}

Se ofreció la participación en el estudio a un total de 79 pacientes que acudieron a la farmacia a retirar hipoglucemiantes orales. 10 (12,7\%) eran cuidadores que venían a recoger la medicación para los enfermos. De los $69(87,3 \%)$ restantes, $5(6,3 \%)$ rechazaron participar por falta de tiempo, por lo que se incluyeron registros de 64 pacientes, $29(45,3 \%)$ mujeres y 35 (54,7\%) hombres.

\section{Datos sociodemográficos}

La edad media en las mujeres fue de 65,5 años (DE=15,3) y en los hombres de 65,6 (DE=12,3). El 75,9\% de las mujeres y el 68,6\% de los hombres eran pensionistas. Los demás datos demográficos se muestran en la tabla 1.

$11(17,2 \%)$ participantes en el estudio además de diabetes padecían otras enfermedades como osteoporosis, cataratas, epilepsia, lupus y depresión, entre otras. 13 (20,3\%) habían sufrido al menos un ingreso hospitalario, de los cuales 2 (3,1\%) fueron debidos a una descompensación diabética.

El número de medicamentos hipoglucemiantes por persona fue de $1,2 \pm 0,5$ (1-4). El de antihipertensivos $0,7 \pm 0,5$ (0-3). El de medicamen-

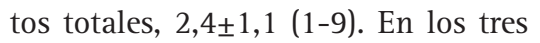
casos $\sin$ diferencias significativas por sexo. Del total de la muestra, 37 $(57,8 \%)$ pacientes estaban en tratamiento con monoterapia y de estos 27 (73,0\%) tomaban metformina, 8 $(21,6 \%)$ iDPP-4 y $2(5,4 \%)$ SGLT2. Se encuentran en tratamiento con doble terapia $25(39,1 \%)$ y sólo $2(3,1 \%)$ están tomando tres fármacos.

\section{Adherencia}

Los resultados de las 8 preguntas del test MMAS-8 se presentan en la tabla 2. La puntuación media fue de $6,2 \pm 2,1$, no encontrándose diferencias

Tabla 1 Datos demográficos de los pacientes

\begin{tabular}{|c|c|c|c|}
\hline & Mujeres n (\%) & Hombres n (\%) & Total n (\%) \\
\hline \multicolumn{4}{|l|}{ Nivel de estudios } \\
\hline Sin estudios & $17(58,6)$ & $9(25,7)$ & $26(40,6)$ \\
\hline Primarios & $8(27,6)$ & $16(45,7)$ & $24(37,5)$ \\
\hline Secundarios/FP & $3(10,3)$ & $7(20,0)$ & $10(15,6)$ \\
\hline Universitarios & $1(3,5)$ & $3(8,6)$ & $4(6,3)$ \\
\hline Total & $29(100)$ & $35(100)$ & $64(100)$ \\
\hline \multicolumn{4}{|l|}{ Enfermedades } \\
\hline Diabetes & $6(20,8)$ & $6(17,0)$ & $12(18,7)$ \\
\hline Diabetes + HTA & $7(24,1)$ & $13(37,1)$ & $20(31,3))$ \\
\hline Diabetes + dislipemia & $5(17,2)$ & $6(17,0)$ & $11(17,2)$ \\
\hline Diabetes + HTA + dislipemia & $11(37,9)$ & $10(28,9)$ & $21(32,8)$ \\
\hline Total & $29(100,0)$ & $35(100,0)$ & $64(100,0)$ \\
\hline \multicolumn{4}{|c|}{ Medicamentos hipoglucemiantes } \\
\hline Metformina & $22(51,2)$ & $28(59,5)$ & $50(78,1)$ \\
\hline IDPP-4 & $11(25,6)$ & $11(23,4)$ & $22(34,4)$ \\
\hline Sulfonilureas & $2(4,6)$ & $2(4,3)$ & $46,3)$ \\
\hline SGLT2 & $1(2,3)$ & $2(4,3)$ & $3(4,7)$ \\
\hline Insulina & $7(16,3)$ & $4(8,5)$ & $11(17,2)$ \\
\hline
\end{tabular}

Tabla 2 Resultados de las preguntas del test MMAS-8 y de percepción sobre el tratamiento

\section{Cuestionario MMAS-8}

1. ¿A veces se le olvida tomar la medicación para la diabetes?

2. En las últimas dos semanas, ¿hubo algún día que se le olvidó tomar la medicación para la diabetes?

3. ¿Alguna vez redujo o no tomó la medicación sin hablar antes con su médico porque se sentía peor cuando la tomaba?

4. Cuando viaja o sale de casa, ¿a veces se le olvida llevar su medicación?

5. ¿Se tomó la medicación para la diabetes ayer?

6. Cuando tiene la glucemia bien controlada ¿deja de tomar su medicación?

7. Tomar la medicación todos los dias es un verdadero inconveniente para algunas personas, ¿alguna vez sintió que fue un inconveniente tomar la medicación para la diabetes?

8. ¿Con qué frecuencia tiene dificultad para acordarse de tomar todos los medicamentos para la diabetes?

\begin{tabular}{|c|c|c|}
\hline $\begin{array}{l}\text { 8. ¿Con qué frecuencia tiene dificultad para acordarse } \\
\text { de tomar todos los medicamentos para la diabetes? }\end{array}$ & & \\
\hline 8.1. Nunca/rara vez & $43(67,2)$ & \\
\hline 8.2. De vez en cuando & $4(6,3)$ & \\
\hline 8.3. A veces & $13(20,3)$ & \\
\hline 8.4. Habitualmente & $4(6,2)$ & \\
\hline 8.5. Todo el tiempo & $0(0,0)$ & \\
\hline 9. ¿Cree que con el tratamiento mejorará su enfermedad? & $54(84,4)$ & $10(15,6)$ \\
\hline $\begin{array}{l}\text { 10. ¿Cree que los medicamentos prescritos son los } \\
\text { adecuados? }\end{array}$ & $59(92,2)$ & $5(7,8)$ \\
\hline
\end{tabular}

* Respuesta correcta.

\section{Sí n (\%) $\quad$ No n (\%)}

$20(31,2) \quad * 44(68,7)$

$16(25,0) \quad *{ }^{*} 48(75,0)$

$14(21,9) \quad *{ }^{*} 50(78,1)$

$11(17,2) \quad *{ }^{*} 53(82,8)$

*53 $(82,8) \quad 11(17,2)$

$6(9,4) \quad *^{*} 58(90,6)$

$22(34,4) \quad * 42(65,6)$

$22(34,4)-42(65,6)$ 
Tabla 3 Adherencia en función del sexo, estudios y situación laboral

\begin{tabular}{|c|c|c|c|c|c|c|c|}
\hline & \multicolumn{2}{|c|}{$\begin{array}{l}\text { Sexo } \\
\text { n (\%) }\end{array}$} & \multicolumn{2}{|c|}{$\begin{array}{l}\text { Estudios } \\
\text { n (\%) }\end{array}$} & \multicolumn{2}{|c|}{$\begin{array}{c}\text { Situación laboral } \\
n(\%)\end{array}$} & \multirow{2}{*}{$\begin{array}{l}\text { Total } \\
\text { n (\%) }\end{array}$} \\
\hline & Mujer & Hombre & $\operatorname{Sin}$ & Con & Pensionista & Activo & \\
\hline $\begin{array}{l}\text { Adherentes } \\
\text { (MMAS-8<6) }\end{array}$ & $19(65,5)$ & $24(68,6)$ & $18(69,2)$ & $25(65,8)$ & $32(69,6)$ & $11(61,1)$ & $43(67,2)$ \\
\hline $\begin{array}{l}\text { No adherentes } \\
\text { (MMAS-8 } \geq 6 \text { ) }\end{array}$ & $10(34,5)$ & $11(31,4)$ & $8(30,8)$ & $13(34,2)$ & $14(30,4)$ & $7(38,9)$ & $21(32,8)$ \\
\hline Total columna & $29(45,3)$ & $35(54,7)$ & $26(40,6)$ & $38(59,4)$ & $46(71,9)$ & $18(28,1)$ & $64(100,0)$ \\
\hline
\end{tabular}

significativas entre sexos. No se encontró relación entre la puntuación del test MMS-8 con respecto a la edad (Rho Spearman $=0,0690 \mathrm{p}=0,5882$ ), ni con el número de medicamentos hipoglucemiantes (Rho de Spearman $=0.0816$ $\mathrm{p}=0.5215$ ), ni tampoco con el número total de medicamentos (Rho Spear$\operatorname{man}=0,0690 \mathrm{p}=0,5882$ ).

El número de pacientes con baja adherencia fue de 21 (32,8\%), con media adherencia $19(29,7 \%)$ y con alta adherencia 24 (37,5\%). Clasificando como adherentes o no a los individuos según la puntuación de corte $<6$ o $\geq 6$, hemos encontrado 43 $(67,2 \%)$ adherentes, siendo más los hombres adherentes que las mujeres $(68,6 \%$ vs $65,5 \%)$, no siendo la diferencia estadísticamente significativa. Lo mismo ocurre con el nivel de estudios y la situación laboral (tabla 3).
Posibles factores relacionados con el nivel de adherencia Sexo

$7(24,1 \%)$ mujeres mostraron una adherencia media, 10 (34,5\%) adherencia baja y $12(41,4 \%)$ alta. En los hombres, 12 (34,3\%) adherencia media, $11(31,4 \%)$ baja y 12 (34,3\%), p-valor $=0,668$, no significativo. El grado de adherencia al tratamiento es independiente del sexo de los pacientes.

\section{Edad}

No se encontró relación entre la puntuación del test MMS-8 con respecto a la edad (Rho Spearman= $0,0690 \mathrm{p}=0,5882$ ).

\section{Nivel de estudios}

La diferencia de puntuación del MMAS-8 en pacientes diabéticos sin estudios respecto a los que tenían es- tudios no fue significativa: $6,2 \pm 2,2$ vs $6,3 \pm 2,0, p$-valor de $W=0,8935$. El porcentaje de adherencia en función del nivel de estudios se muestra en la figura 3. En ella se observa que los universitarios tienen, en su mayoría (75\%), media adherencia y el 41,7\% de personas con estudios primarios muestran una adherencia baja al tratamiento. La prueba estadística da un p-valor=0,355, no significativo. El grado de adherencia al tratamiento es independiente del nivel de estudios de los pacientes.

\section{Condición laboral}

La relación entre la condición laboral y el grado de adherencia se presenta en la figura 4. La prueba estadística da un $\mathrm{p}$-valor $=0,5989$, no significativo. El grado de adherencia al tratamiento es independiente de la condición laboral de los pacientes.

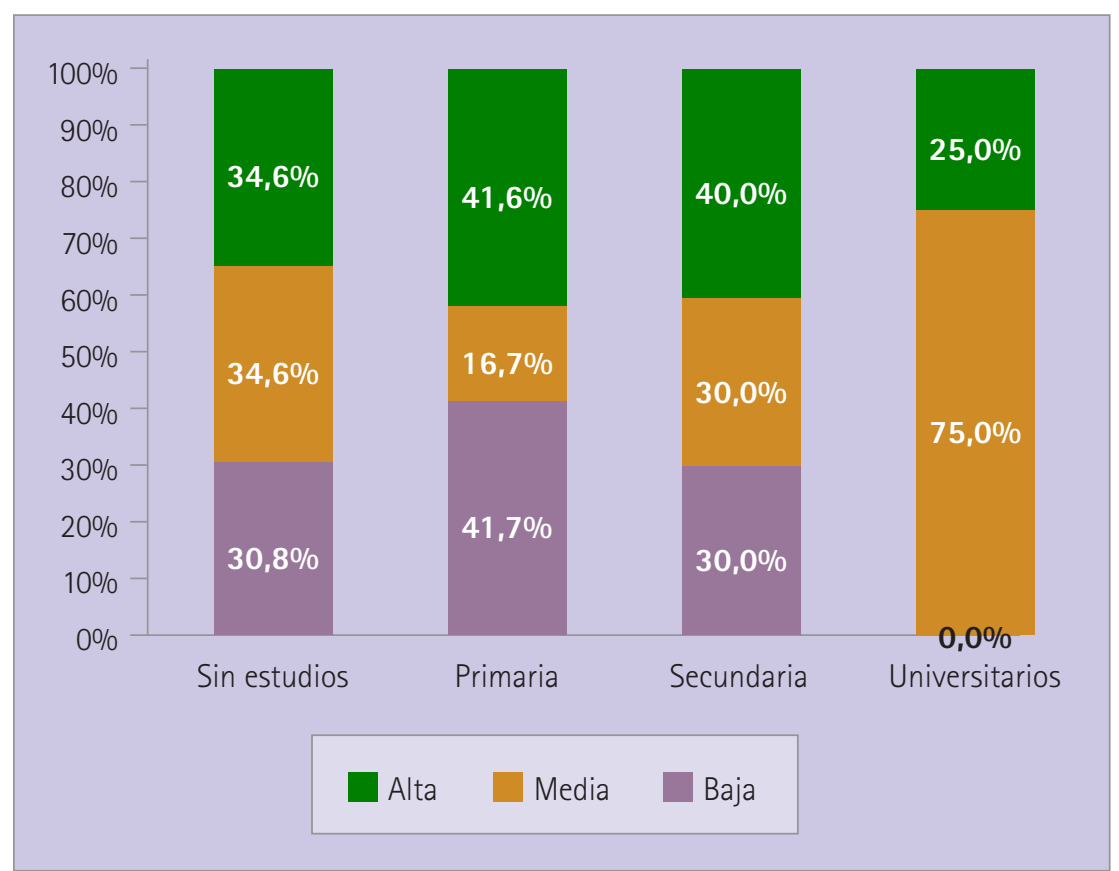

Figura 3 Nivel de estudios y adherencia

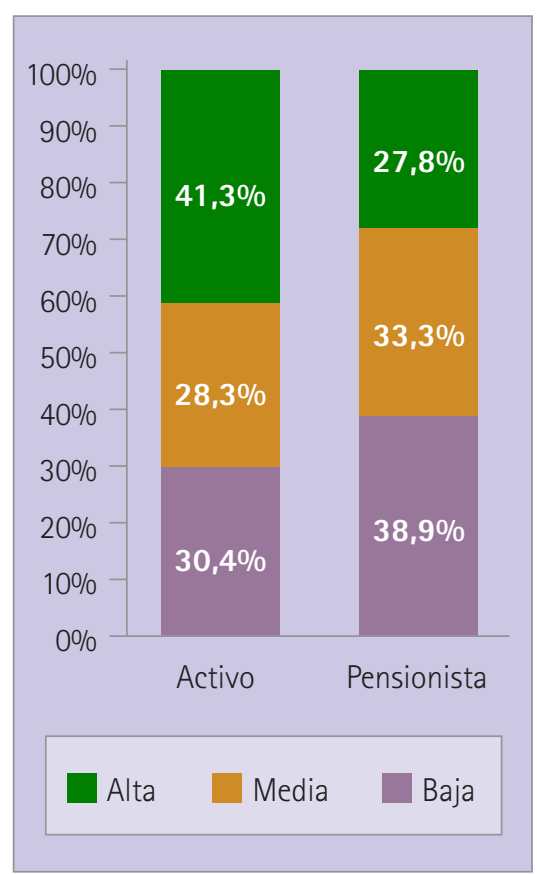

Figura 4 Adherencia según condición laboral 
Percepción de los pacientes sobre su tratamiento y su posible relación con el porcentaje de adherencia

$10(15,6 \%)$ de los pacientes con diabetes no creen que el tratamiento mejore su enfermedad y $5(7,8 \%)$ no creen que los medicamentos que tienen prescritos sean los adecuados.

La creencia de que el tratamiento mejora la enfermedad aumenta la adherencia. La puntuación del MMAS8 fue de $6,5 \pm 1,9$ vs $4,9 \pm 2,4$ con un p-valor de $W=0,0147$. La creencia de que los medicamentos prescritos son los adecuados aumenta la adherencia, aunque no de forma estadísticamente significativa. La puntuación del MMAS- 8 fue $6,3 \pm 2,8$ vs $5,5 \pm 2,2$ con un p-valor de $\mathrm{W}=0,1248$.

La creencia de que el tratamiento mejora la enfermedad, y que los medicamentos prescritos son los adecuados (ambas), aumenta la adherencia. La puntuación MMAS- 8 fue $6,6 \pm 1,8$ vs $4,6 \pm 2,5$ con un $p$-valor de $W=0,0036$.

\section{Discusión}

El hecho de tratarse de un estudio realizado en una sola farmacia ha sido la causa de no haber logrado el tamaño de muestra esperado. De las 79 personas que solicitaban la dispensación de un medicamento antidiabético a los que se ofreció participar, 5 no aceptaron y en 10 casos quien acudía a la farmacia era un cuidador al que, por tanto, no podíamos entrevistar. $\mathrm{Al}$ final, 64 personas aceptaron y cumplimentaron el cuestionario válido. No obstante, consideramos que el pilotaje ha resultado positivo y que sería muy interesante continuar con el uso del cuestionario Moriski-Green-Levine modificado, MMAS-8 en futuros estudios en FC, ya que aporta al estudio de la adherencia elementos hasta ahora no analizados como la definición de grados de cumplimiento: baja, media o alta.

\section{Características sociodemográficas de la muestra}

Los pacientes son mayoritariamente hombres de edad adulta mayores de 60 años, con hipertensión y dislipemias, características propias de las personas con diabetes e incluidas en la mayoría de los estudios revisados a nivel nacional $[4,7,17,18,22-24]$ y también se encuentran estos datos en el último estudio realizado en Galicia [9], con un porcentaje de 49\% de mujeres y 51\% de hombres.

La prevalencia de hipertensión arterial que encontramos en estos pacientes es elevada, 31,3\% frente al 18,2\%, que solamente padecen diabetes. Coincide con la mayoría de los estudios $[22,23]$. Lo que confirma a la hipertensión como la enfermedad o complicación más importante de los diabéticos después de la hiperglucemia.

Otro factor de riesgo cardiovascular que se encontró fue la dislipemia, al igual que en otros estudios [24]. Después de la hipertensión, es el más frecuente en la población diabética, situación que corresponde al síndrome metabólico, asociado a hábitos alimenticios inadecuados, vida sedentaria, elevado índice de masa corporal, tabaquismo etc.

\section{Utilización de medicamentos}

En lo que respecta al tipo de medicamentos hipoglucemiantes utilizados por los individuos de nuestro estudio, metformina es el más empleado $(78,1 \%)$, coincidiendo con todos los estudios revisados [24] y tal como se recomienda en las guías clínicas de referencia. El segundo grupo de medicamentos hipoglucemiantes fueron los inhibidores de la dipeptidyl peptidasa 4 (IDPP-4), seguidos de la insulina. En el estudio de Fornos et al [24] el segundo porcentaje mayor corresponde con las sulfonilureas. Esto se corresponde con las guias de consenso para el tratamiento del paciente diabético tipo 2, en las cuales se recomienda como primera línea de tratamiento metformina y si los objetivos no son alcanzados en 3 meses, se pasaría a combinar con una sulfonilurea o un iDPP-4 u otros $[25,26]$.

En cuanto al número de hipoglucemiantes utilizados, los pacientes en régimen de monoterapia con metformina representan el 57,8\% del total de la muestra, al igual que en el estudio de Boccuzzi et al [27]. Esto haría pensar que la mayoría de los pacientes tienen un buen control de su enfermedad siendo innecesaria la doble o triple terapia, o bien que están en los inicios de la enfermedad, pregunta que se recogió en el cuestionario. Sin embargo, la mayoría de los pacientes no sabían exactamente el inicio de la enfermedad, por ello, fue una variable que no se pudo valorar.

Posibles datos de adherencia en relación el test MMAS-8

La puntuación media del test de MMAS-8 fue de 6,2 sin diferencias estadísticamente significativas entre sexos, edad, número de hipoglucemiantes o el total de medicamentos. Este valor fue ligeramente inferior al encontrado en el trabajo de Dhippayom [28], de características similares al nuestro, en el que la puntuación media del test fue de 6,8.

La dificultad que hemos encontrado para comparar nuestros resultados con otros estudios fue que la mayor parte de ellos utilizan el test de Morisky-Green de 4 ítems, clasificando a los pacientes como cumplidores e incumplidores. En la mayoría de los estudios encontrados que utilicen el nuevo test no se señalan valores medios de la puntuación del test MMAS8, pero sí establecen el punto de corte en 6 , de modo que consideran no adherentes a los pacientes con diabetes que dan una puntuación en el test $<6$. Nosotros encontramos un 32,8\% de individuos con baja adherencia $(<6)$ o no adherentes según ese criterio. Valor similar al de Wong et al $(32,2 \%)$ [29]; mayor al encontrado por Fadare et al (26,6\%) [30] y por Al-Haj Mohd et al $(26,5 \%)$ [31], e inferior a los encontrados por Jamous et al $(42,7 \%)$ [32], Dhippayom et al (64,6\%) [28] y Chew et al $(43,0 \%)$ [33].

\section{Otros posibles factores}

relacionados con el nivel de adherencia

Los factores que se han valorado con posible relación con el grado de adherencia al tratamiento son el sexo, la edad, el nivel de estudios y la condición laboral (pensionista/activo). Aunque las diferencias entre grupos no resultaron estadísticamente significativas, por lo que no habría relación entre estas variables y el porcentaje de adherencia a los tratamientos farmacológicos, algunas tenían cierta importancia que queremos resaltar.

Un porcentaje elevado de mujeres demostraron una adherencia clasificada como alta al tratamiento, mayor con respecto a los hombres, al contrario que en el estudio [9] y en el que, 
además, la diferencia fue significativa para el test de Haynes-Sacket, otro método indirecto con el que se mide el nivel de cumplimiento y adherencia al tratamiento. Esta comparación hay que valorarla con cautela, por la diferencia de test utilizados.

En varios estudios se ha observado que el nivel escolar facilita la adherencia al tratamiento porque los pacientes son capaces de comprender y tomar mayor conciencia sobre su estado. La prevalencia de Diabetes Mellitus tipo 2 parece relacionarse con el nivel de estudios y la situación socioeconómica. En la Encuesta Nacional de Salud de 2017 se encuentra una relación inversa entre la prevalencia de DM2 y el nivel de estudios [34]. Por lo tanto, el nivel de estudios sería un factor de riesgo a considerar como predictor de la prevalencia de DM2.

En el estudio más cercano en el tiempo y lugar, Fornos et al [9] concluyen que la adherencia disminuye a menor nivel de estudios, al igual que todos los estudios revisados hasta el día de hoy. A este respecto, en el nuestro, al relacionar las personas con estudios (primarios, secundarios o universitarios) y sin estudios con la adherencia, no encontramos diferencias significativas entre estos dos parámetros. Es probable que la razón sea, además de la pequeña muestra, la diferencia en el cuestionario. Convendría, por tanto, continuar con la utilización del mismo en farmacia comunitaria, con una muestra mucho más amplia, para corroborar o no dicha diferencia.

En cuanto al porcentaje de adherencia según la condición laboral (pensionista/activo), encontramos que en los pensionistas es notablemente mayor el número de adherentes que en la población activa. Esto puede ser debido a la falta de tiempo en la población trabajadora que les impide poder ir a la farmacia a por los medicamentos e incluso en los cambios de horarios a los que están sometidos en el trabajo. Los pacientes pensionistas, y por lo tanto los de mayor edad, en el estudio de Fornos et al [9] presentan ser más incumplidores de manera significativa para los test de Batalla y Haynes-Sacket pero, sin embargo, no para el test de Morisky-Green. Esta diferencia con respecto a nuestros resultados puede ser debida a la diferencia de tamaño de la muestra entre nuestro estudio, 64 personas, y el CumpleGA [9], 1.588 personas.
Percepción de los pacientes sobre su tratamiento/enfermedad y su posible relación con la adherencia

En cuanto a la percepción que tienen los pacientes sobre su medicación, el $84,4 \%$ cree que con el tratamiento mejorará su enfermedad y el 92,2\% cree que los medicamentos que tiene prescritos son los adecuados. Cuando relacionamos estos parámetros con el porcentaje de adherencia, los datos muestran que la confianza en que el tratamiento mejora la enfermedad y que los medicamentos prescritos son los adecuados, aumenta la adherencia, siendo el resultado del MMAS-8 dos puntos más elevado con un p-valor de 0,0036. En el caso del estudio realizado a nivel nacional por Fornos et al [9], se encontró un porcentaje del $82,7 \%$ de respuestas positivas en la pregunta 9 y un 70,5\% en la pregunta 10. En cuanto a la relación de estas respuestas con el porcentaje de adherencia [9], lo hace en relación con el cumplimiento terapéutico, obteniendo también que la prevalencia del incumplimiento resulta menor entre los pacientes que tienen una percepción positiva de sus tratamientos. Comprobamos, por tanto, que coincidimos con ese estudio en la importancia del empoderamiento de los pacientes para mejorar su adherencia terapéutica.

Como conclusión general consideramos que la farmacia comunitaria es un centro sanitario adecuado para la evaluación de la adherencia del tratamiento con este tipo de métodos y la intervención del farmacéutico es fundamental para mejorar dicha adherencia.

\section{Conclusiones}

- El cuestionario MMAS-8 se ha mostrado como una herramienta útil en la farmacia comunitaria para la evaluación de la adherencia terapéutica por parte de los farmacéuticos investigadores.

- El porcentaje de adherentes resultó superior al 65\%. Presentando el 32,8\% baja adherencia, 29,7\% media y sólo el 37,5\% restante alta adherencia al tratamiento hipoglucemiante.

- No se encontró relación entre el porcentaje de adherencia obtenido en el test con los factores: sexo, edad, nivel de estudios y situación laboral.
- Casi el 85\% de los pacientes tiene una percepción positiva acerca de que su tratamiento mejorará la enfermedad. La creencia de que el tratamiento mejora la enfermedad y que los medicamentos prescritos son los adecuados aumenta la adherencia.

\section{Referencias bibliográficas}

1. World Health Organization. Adherence to long-term therapies. Evidence for action. Geneve: WHO; 2003.

2. Foro de Atención Farmacéutica en Farmacia Comunitaria. Guía práctica para los Servicios de Atención Farmacéutica en la Farmacia Comunitaria. Madrid: Consejo General de Colegios Oficiales de Farmacéuticos; 2010.

3. García Jiménez E. Incumplimiento como causa de problemas relacionado con medicamentos en el seguimiento farmacoterapéutico [Tesis doctoral]. Granada: Universidad de Granada; 2003.

4. García-Jiménez E, Amariles P, Machuca M, Parras-Martín M, Espejo-Guerrero J, Faus MJ. Incumplimiento, problemas relacionados con los medicamentos y resultados negativos asociados a la medicación: causas y resultados en el seguimiento farmacoterapéutico. Ars Pharm. 2008: 49(2):145-157.

5. Orueta R, Toledano P, Gómez-Calcerrada RM. Actualización en Medicina de Familia: Cumplimiento terapéutico. SEMERGEN. 2008; 34(5):235-43. doi:10.1016/S1138-3593(08)71889-6

6. Sanahuja MA, Villagrasa V, Martinez-Romero F. Adherencia Terapéutica. Pharm Care Esp. 2012; 14(4):162167.

7. Escobar C, Divisón JA. Importancia de la adherencia al tratamiento. SEMERGEN. 2017; 43(3):236-237. doi:10.1016/j.semerg.2016.04.021

8. Dilla T, Valladares A, Lizán L, Sacristán JA. Adherencia y persistencia terapéutica: causas, consecuencias y estrategias de mejora. Aten Primaria. 2009; 41(6):342-348. doi:10.1016/j. aprim.2008.09.031

9. Fornos-Pérez JA, Andrés-Rodriguez NF, Andrés-Iglesias JC, Mera-Gallego R, Mera-Gallego I, Penín-Álvarez 0 , et al. Valoración del cumplimiento de los tratamientos hipoglucemiantes y antihipertensivos en Galicia (CumpleGA). Farmacéuticos Comunitarios 2017; 9(4):5-13. doi:10.5672/ FC.2173-9218.(2017/Vol9).004.02

10. Vilaplana-Pérez C, González-Javier F, Ordoñana-Martín JR. Adherencia al tratamiento. Una revisión desde la perspectiva farmacéutica. Pharm Care Esp. 2012; 14(6):249-255. 
11. Pinto JL, Fernández de Cano-Martín $\mathrm{N}$, Ollero $\mathrm{M}$, Barragán $\mathrm{B}$, Aliaga $\mathrm{A}$ González-Jurado M. Documento de Consenso. Una aproximación multidisciplinar al problema de la adherencia terapéutica en las enfermedades crónicas: estado de la situación $\mathrm{y}$ perspectivas de futuro. Madrid: DGMM; 2012.

12. Orozco-Beltrán D, Mata-Cases M, Artola S, Conthe P, Mediavilla J, Miranda C. Abordaje de la adherencia en diabetes mellitus tipo 2: situación actual y propuesta de posibles soluciones. Aten Primaria. 2016: 8(6):406420. doi:10.1016/j.aprim.2015.09.001

13. Pousinho S, Morgado M, Falcao A, Alves G. Pharmacist Interventions in the Management of Type 2 Diabetes Mellitus: A Systematic Review of Randomized Controlled Trials. J Manag Care Spec Pharm. 2016; 22(5):493-515. doi:10.18553/ jmcp.2016.22.5.493

14. SEFAC y Sociedad Española de Diabetes. Guía Práctica de Atención Farmacéutica al Paciente Diabético. Madrid: SEFAC; 2015. ISBM: 978-84615-9039-1.

15. Kirkman MS, Rowan-Martin MT, Levin R, Fonseca VA, Schmittdiel JA, Herman WH, Aubert RE. Determinants of Adherence to Diabetes Medications: Findings from a Large Pharmacy Claims Database. Diabetes Care 2015; 38:604-609. doi:10.2337/ dc14-2098

16. Capoccia K, Odegard PS, Letassy N. Medication Adherence with Diabetes Medication. A Systematic Review of the Literature. The Diabetes Educator. 2016; 42(1):34-71. doi:10.1177/0145721715619038

17. Cramer JA. A Systematic Review of Adherence with Medications for Diabetes. Diabetes Care 2004: 27(5):12181224. doi:10.2337/diacare.27.5.1218

18. Gutiérrez-Angulo ML, Lopetegui-Uranga P, Sánchez-Martín I, Garaigordóbil-Landazabal M. Cumplimiento terapéutico en pacientes con hipertensión arterial y diabetes mellitus 2. Rev Calid Asist. 2012; 27(2):7277. doi:10.1016/j.cali.2011.09.008
19. Carbonell C, Guañabens N, Regadera L, Marín JA, Taverna E, Ayechu MP. Análisis del cumplimiento terapéutico en mujeres con osteoporosis. Reumatol Clin. 2011; 7(5):299-304. doi:10.1016/j.reuma.2010.12.003

20. Martínez-Pérez P, Pomares-Gómez F, Orozco-Beltrán D, Carratalá-Munuera MC, Quesada-Rico JA, Mira-Solves JJ. Validación de la Morisky Medication Adherence Scale 8-items (MMAS-8) para el tratamiento de la Diabetes Mellitus Tipo 2 (DM2) en España. Endocrinol Nutri. 2017; 64(1):43.

21. Soriguer F, Goday A, Bosch-Comas A, Bordiu E, Calle-Pascual A, Carmena R et al. Prevalence of diabetes mellitus and impaired glucose regulation in Spain: the Di@bet.es Study. Diabetologia 2012; 55:88-93. doi:10.1007/ s00125-011-2336-9

22. Figueiredo IV, Moteiro C, Castel-Branco MM, Caramona MM, Fernández-Llimós F. Seguimiento de pacientes con diabetes tipo 2 en una unidad de atención primaria de salud. Pharm Care Esp. 2014; 16(2):39-48.

23. Maidana GM, Mastroianni PC, Vera Z, Samaniego L, Acosta P, Lugo GB. Impacto de la atención farmacéutica en los resultados clínicos y en la calidad de vida de pacientes con diabetes mellitus tipo 2. Pharm Care Esp. 2016; 18(3):107-121.

24. Fornos JA, García GP, Fernández M, González D, Andrés NF, Andrés JC. Evaluación del conocimiento, el cumplimiento y la satisfacción del tratamiento en pacientes diabéticos en la oficina de farmacia. Avances en Diabetología 2008; 24(5):399-406.

25. RedGDPS. Práctica clínica en la DM2. Análisis crítico de las evidencias por la RedGDPS: Elsevier; 2011.

26. Gómez-Peralta F, Escalada-Sanmartín J, Menéndez-Torre E, Mata-Cases M, Ferrer-García JC, Ezkurra-Loiola $\mathrm{P}$, et al. Recomendaciones de la Sociedad Española de Diabetes (SED) para el tratamiento farmacológico de la hiperglucemia en la diabetes tipo 2: Actualización 2018. Endocrinol Diabetes Nutr. 2018; 65(10):611-624. doi:10.1016/j.endinu.2018.08.004
27. Boccuzzi SJ, Wogen J, Fox J, Sung JCY. Shah AB, Kim J: Utilization of oral hypoglycemic agents in a drug-insured U.S. population. Diabetes Care. 2001; 24:1411-1415. doi:10.2337/diacare.24.8.1411

28. Dhippayom T, Krass I. Medication-taking behaviour in New South Wales patients with type 2 diabetes: an observational study. Aust J Prim Health. 2015; 21(4):429-437. doi:10.1071/PY14062

29. Wong MC, Wu CH, Wang HH, Li HW, Hui EM, Lam AT, et al. Association between the 8-item Morisky medication adherence scale (MMAS-8) score and glycaemic control among Chinese diabetes patients. J Clin Pharmacol. 2015; 55(3):279-87. doi:10.1002/ jcph.408

30. Fadare J, Olamoyegun M, Gbadegesin BA. Medication adherence and direct treatment cost among diabetes patients attending a tertiary healthcare facility in Ogbomosho, Nigeria. Malawi Med J. 2015; 27(2):65-70. doi:10.4314/mmj.v27i2.7

31. Al-Haj Mohd MM, Phung H, Sun J, Morisky DE. Improving adherence to medication in adults with diabetes in the United Arab Emirates. BMC Public Health. 2016; 16(1):857. doi:10.1186/ s12889-016-3492-0

32. Jamous RM, Sweileh WM, Abu-Taha AS, Sawalha AF, Zyoud SH, Morisky DE. Adherence and satisfaction with oral hypoglycemic medications: a pilot study in Palestine. Int J Clin Pharm. 2011; 33(6):942-948. doi:10.1007/s11096-011-9561-7

33. Chew BH, Hassan NH, Sherina MS. Determinants of medication adherence among adults with type 2 diabetes mellitus in three Malaysian public health clinics: a cross-sectional study. Patient Prefer Adherence. 2015; 6(9):639-48. doi:10.2147/PPA.S81612

34. Ministerio de Sanidad, Consumo y Bienestar Social [Internet]. Encuesta Nacional de Salud de España 2017. [Acceso 12/1/2019]. Disponible en: https://www.mscbs.gob.es/estadEstudios/estadisticas/encuestaNacional/ encuestaNac2017/ENSE17_MOD1_ REL.pdf 